\title{
Conduction through demyelinated plaques in multiple sclerosis: computer simulations of facilitation by short internodes
}

\author{
STEPHEN G. WAXMAN, A ND MICHAEL H. BRILL \\ From the Department of Neurology, Harvard Medical School, Beth Israel Hospital, Boston; \\ Harvard-MIT Division of Health Sciences and Technology, and Research Laboratory of Electronics, \\ Massachusetts Institute of Technology, Cambridge, Massachusetts, USA
}

S U M MARY Clinical and laboratory observations both suggest that it may be possible for action potentials to traverse, in a continuous manner and without interruption, demyelinated zones along some axons. This continuous mode of conduction requires the presence of sufficient numbers of sodium channels in the demyelinated region. One of the factors which will tend to prevent such conduction is the impedance mismatch at sites of focal demyelination, which may result in a reduction in current density sufficient to cause conduction failure. As part of an effort to examine the conditions which would promote conduction into, and beyond, the demyelinated region, we examined, using computer simulations, the effects of reduction in length of the proximal internodes closest to the demyelinated region. Our results indicate that reduction in length of the two internodes closest to the demyelinated region, to approximately one-third of normal length or less, will facilitate conduction beyond the plaque. The results suggest that reductions in internode length, which have been histologically observed along some demyelinated fibres, may have functional significance in terms of facilitating conduction past focally demyelinated zones.

An important problem in the pathophysiology of multiple sclerosis concerns the prerequisites for axonal conduction through demyelinated plaques. Clinical and experimental observations both suggest that, under some conditions, conduction may proceed without interruption, albeit at a reduced conduction velocity, through regions of total demyelination. Pathological studies, for example, have demonstrated that some demyelinated plaques may be asymptomatic (Ghatak et al., 1974), and that the distribution of plaques may be more widespread than would be predicted from the clinical deficits (Namerow and Thompson, 1969). One implication of these observations is that conduction may be preserved along at least some of the demyelinated axons. Clinical neurophysiological observations are consistent with this hypothesis. Visual evoked potentials, for example, may be delayed by more than $40 \mathrm{~ms}$ in multiple sclerosis patients with no history of optic neuritis

Address for reprint requests: Dr Stephen G. Waxman, Harvard Neurological Unit, Beth Israel Hospital, 330 Brookline Avenue, Boston, Mass. 02215, USA.

Accepted 22 November 1977
(Halliday et al., 1973). Finally, it has recently been shown experimentally that impulse conduction may occur in a continuous fashion along demyelinated regions as long as $500 \mu \mathrm{m}$ in fibres exposed to diphtheria toxin (Bostock and Sears, 1976). These observations bring up an important question in the physiology of demyelinated fibres. Recent data suggest that in normal fibres, sodium channel density at nodes of Ranvier $\left(12000 / \mu \mathrm{m}^{2}\right)$ is much higher than in the internodal axon membrane beneath the myelin $\left(<25 / \mu \mathrm{m}^{2}\right.$; Ritchie and Rogart, 1977). On the other hand, the observations of conduction past regions of focal demyelination imply the existence of a sufficient density of sodium channels to support conduction in the demyelinated region of some fibres, and it has been suggested that sodium channel densities may change after demyelination (Ritchie and Rogart, 1977; Waxman, 1977). However, computer simulations (Waxman, 1977) have demonstrated that the presence of a high density of sodium channels in the demyelinated region may not, in itself, ensure conduction past the site of demyelination. For example, conduction failure may occur at a single focally 
demyelinated internode, even if ionic channel densities are as high in the demyelinated region as at normal nodes of Ranvier, as a result of impedance mismatch between the normal and demyelinated regions. Conduction block will occur as a result of failure of initiation of activity in the demyelinated region, if current density in the demyelinated area is sufficiently small because of the increased surface area of bared axonal membrane in the demyelinated zone. Thinning of myelin in areas adjacent to the bared region may contribute to current loss. The situation is similar to that at other regions of impedance mismatch-for example, at sites of axial inhomogeneity in non-myelinated axons (Khodorov et al., 1969; Ramon et al., 1975; Parnas et al., 1976) and at the junction between neuronal cell body and initial segment (Dodge and Cooley, 1973), where safety factor is low and where conduction failure has been experimentally observed.

The elegant studies of Rasminsky and Sears (1972), which have been followed by the investigations of Bostock and Sears (1976) and Rasminsky et al. (1977), have examined the physiology of conduction along abnormally myelinated fibres. The latter two studies provide experimental evidence for continuous conduction along portions of some demyelinated and amyelinated axons. The earlier computer simulation studies of Koles and Rasminsky (1972) were important in delineating some of the biophysical bases of abnormal conduction in demyelinated fibres, and were based on the assumption that membrane excitability remains confined to the nodes in demyelinated axons. As part of a programme to explore further the pathophysiology of multiple sclerosis and of mechanisms which might promote impulse conduction past demyelinated plaques, we have studied, using computer simulations, several possible mechanisms for continuous conduction along focally demyelinated axons. In this paper we present results which suggest that reduction in internode length proximal to a demyelinated region may promote conduction of impulses into, and past, that region. As noted in the Discussion, there is experimental evidence for reductions in internode distance along axons which have been subjected to demyelination.

\section{Methods}

Methods used in the present study were adapted from those of Brill et al. (1977) and involve the numerical integration of the equation

$$
c(x)-\frac{\partial}{\partial t} V(x, t)=\frac{1}{r_{a}} \frac{\partial^{2}}{\partial x^{2}} V(x, t)-i_{m}(x, t)
$$

where:

$\mathrm{V}$ is the potential across either the nodal membrane or myelin. $r_{a}$ is the axial resistance of the fibre per unit length.

$c(x)$ is the capacitance per unit length-that is, trans-myelin capacitance $\mathrm{C}_{M}$ in the internodal region, and the nodal and demyelinated axolemma capacitance $C_{N}$ at nodes of Ranvier and demyelinated regions.

$i_{m}$ is the ionic current per unit length of membrane.

In the internodal regions,

$$
i_{m}(x, t)=g_{M} V(x, t)
$$

where $g_{M}$ is the myelin conductance per unit length. At each node $\mathrm{j}$,

$$
\mathrm{i}_{\mathrm{m}}(\mathrm{x}, \mathrm{t})=\mathrm{I}_{\mathrm{H} \text { H. }} \pi \mathrm{d} \cdot \frac{\mathrm{NL}}{\Delta \mathrm{x}}+\frac{\Delta \mathrm{x}-\mathrm{NL}}{\Delta \mathrm{x}}\left[\mathrm{V}_{\mathrm{j} \cdot \mathrm{g}_{\mathrm{M}}}\right]
$$

where the current density $I_{H H}$ is given by

$I_{H H}=\bar{g}_{\mathrm{Na}} \mathrm{m}_{j}{ }^{3} \mathrm{~h}_{j}\left[\mathrm{~V}_{\mathrm{j}}-\mathrm{V}_{\mathrm{Na}}\right]+\overline{\mathbf{g}}_{\mathrm{K}} \mathrm{n}_{\mathrm{j}}{ }^{4}\left[\mathrm{~V}_{\mathrm{j}}-\mathrm{V}_{\mathrm{K}}\right]+$

$$
\mathrm{g}_{\mathrm{L}}\left[\mathrm{V}_{\mathrm{j}}-\mathrm{V}_{\mathrm{L}}\right]
$$

where $m_{j}, n_{j}$, and $h_{j}$ satisfy the usual differential equations in time (Hodgkin and Huxley, 1952; Fitzhugh, 1962).

Equations were integrated numerically by the Crank-Nicholson method implemented on a PDP 9 computer, as described for unmyelinated fibres by Moore et al. (1975) and adapted for the myelinated fibre by R. W. Joyner.

We used an integration time increment $\Delta \mathrm{t}=5 \mu \mathrm{S}$ and a length increment $\Delta \mathrm{x}=200 \mu \mathrm{m}$. Thus there were 10 spatial lumped-parameter segments per normal internode length $(\mathrm{L}=2000 \mu \mathrm{m})$. We composed each of our simulated fibres out of three kinds of segments (see Table for parameter values): (i) segments with the electrical properties of normal myelin; (ii) segments containing a node of Ranvier, with lumped electrical parameters from the node and its adjacent myelin; for length increment $\Delta \mathrm{x}$ and nodal length NL, the capacitance assigned to a segment containing a node is $\mathrm{C}^{*}=\frac{\mathrm{C}_{\mathrm{N}} \mathrm{NL}+\mathrm{C}_{\mathrm{m}}(\Delta \mathrm{x}-\mathrm{NL})}{\Delta \mathrm{x}}$; (iii) segments consisting of active membrane with no adjacent myelin. These segments comprise the demyelinated region of the fibre. We allowed the active membrane to be normal nodal membrane (Table; see also Brill et al., 1977) or Hodgkin-Huxley membrane (Table), and observed the effect on conduction of each of these alternatives.

For a normal fibre (without focal demyelination) every tenth segment contains a node (type ii segment), and the rest are myelin (type i). We modelled focal demyelination by replacing the segment containing the fourth node with a myelin segment, and by replacing the following internode and node with 10 type iii segments. This left a region of non-myelinated axon $\left(D_{1}-D_{4}\right)$ equal in length to one normal internode. Distal to this demyelinated region the fibre was 
Table Parameters

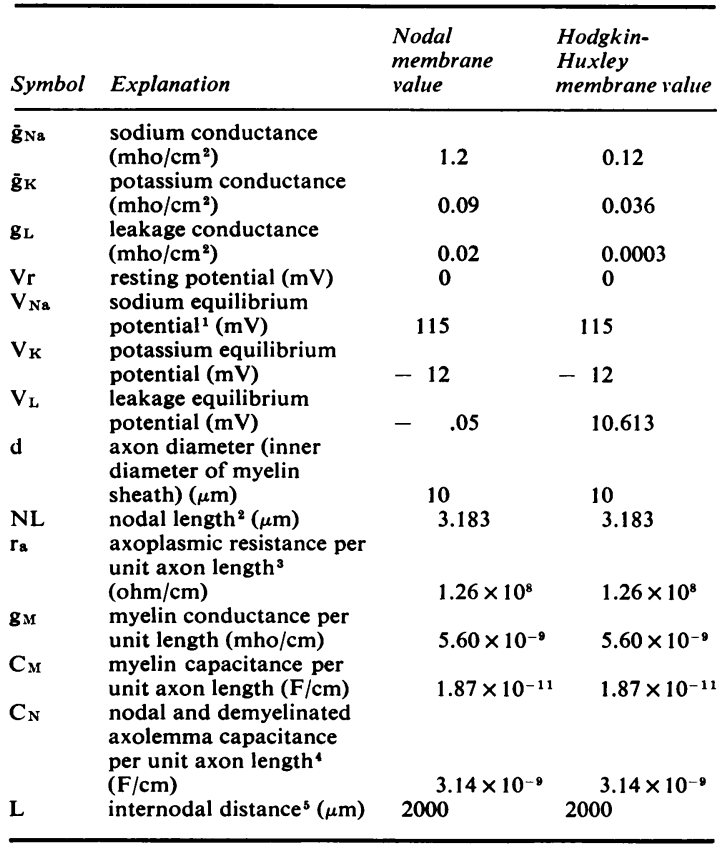

${ }^{1}$ All voltage signs are reversed from those of the original HodgkinHuxley formulation.

${ }^{2}$ Calculated from nodal area of $100 \mu \mathrm{m}^{2}$

${ }^{3}$ Calculated from specific axoplasmic resistance of $100 \mathrm{ohm}-\mathrm{cm}$.

Calculated from capacitance per unit area of $10^{-6} \mathrm{~F} / \mathrm{cm}^{2}$.

${ }^{8}$ Except proximal to demyelinated regions as indicated in text.

composed of normal internodes and nodes. We designated the three nodes proximal to the demyelinated region " 1, ," " 2 ," and " 3 ," and the nodes distal to the demyelinated region as " $4, "$ " 5 ," " 6 ," etc. Simulated fibres were 11 nodes in length, although only the demyelinated region and first six nodes are shown in the Figures.

Fibres were examined with either normal nodal membrane in the demyelinated region (Figs. 1-5) or with Hodgkin-Huxley membrane in this region (Fig. 6). We examined the conduction properties of each of these fibres by stimulating with a twicethreshold current for $200 \mu$ s at the beginning of the internode before node 1 . Conduction delays were measured by noting the time differences for the first $50 \mathrm{mV}$ crossings of action potentials at designated points along the fibre. Rate constants were adjusted to $20^{\circ} \mathrm{C}$.

In order to model the effect of interposing short internodes proximal to the demyelinated region, we replaced one of the myelin segments between node 3 and the demyelinated region $\left(D_{1}-D_{4}\right)$ with a nodal (type ii) segment. Figure 2 shows a representative case : the demyelinated region is made of normal nodal membrane, and the new node partitions the preceding internode into two internodes, one with length $1600 \mu \mathrm{m}$ and one with length $400 \mu \mathrm{m}$. We also examined the effect of replacing, with type ii segments, $t w o$ of the internodal segments between node 3 and the demyelinated region, in order to interpose two short internodes proximal to the demyelinated zone. Representative cases are shown in Figs. 3 to 6. In all Figures, the new nodes are labelled $A$ and $B$, and the first, fourth, seventh, and tenth segments of the demyelinated region are labelled $D_{1}-D_{4}$. Internodes were designated by reference to their endpoints: thus, internode 2-3 extends between node 2 and node 3 , and internode $A-D_{1}$ extends between node $A$ and point $\mathbf{D}_{1}$.

\section{Results}

The effect of focal demyelination at a single internode is shown in Fig. 1. In the fibre represented, internodes 1-2 (the internode between 1 and 2), 2-3, 3- $D_{1}, D_{4}-4$, $4-5,5-6$, and all subsequent internodes are normally myelinated (see Table). The internode between points $D_{1}$ and $D_{4}$ is focally demyelinated, with nodal specific membrane properties throughout the demyelinated region. Internodal conduction time proximal to the site of demyelination was $102 \mu$ s (conduction velocity $=19.7 \mathrm{~m} / \mathrm{s}$ ), as in simulations of normally myelinated fibres. Despite the assumption of nodal membrane properties in the demyelinated region, conduction failure occurs, and the spike does not invade the demyelinated zone. This failure does not reflect inexcitability of the demyelinated axon membrane, but rather reflects the low current density in this region.

Figure 2 shows the effect of introducing a single internode, substantially reduced in length $(400 \mu \mathrm{m})$ just proximal to the site of demyelination. Internode 3- $\mathrm{D}_{1}$ (originally $2000 \mu \mathrm{m}$ in length) is now replaced by internode 3-A $(1600 \mu \mathrm{m})$, node $A$, and internode $A-D_{1}(400 \mu \mathrm{m})$. Excitation of node $A$ is delayed (internodal conduction time for $3-\mathrm{A}$ is $0.19 \mathrm{~ms}$, compared to a normal internodal conduction time of $0.102 \mathrm{~ms}$ ), and the impulse does not invade the demyelinated region. A similar simulation, in which internode $A-D_{1}$ was made $200 \mu \mathrm{m}$ long, also resulted in conduction failure.

Figure 3 shows the effect of two closely spaced nodes (nodes A, B; internode lengths A-B and B-D $=200 \mu \mathrm{m})$ located immediately proximal to the demyelinated area. In contrast to the simulations shown previously, in this case conduction proceeds through the demyelinated zone. The impulse invades the demyelinated region $\left(D_{1}-D_{4}\right)$, propagates at a conduction velocity close to the steady-state value of $1.64 \mathrm{~m} / \mathrm{s}$ observed in simulations of uniform nonmyelinated fibres with nodal membrane properties, 


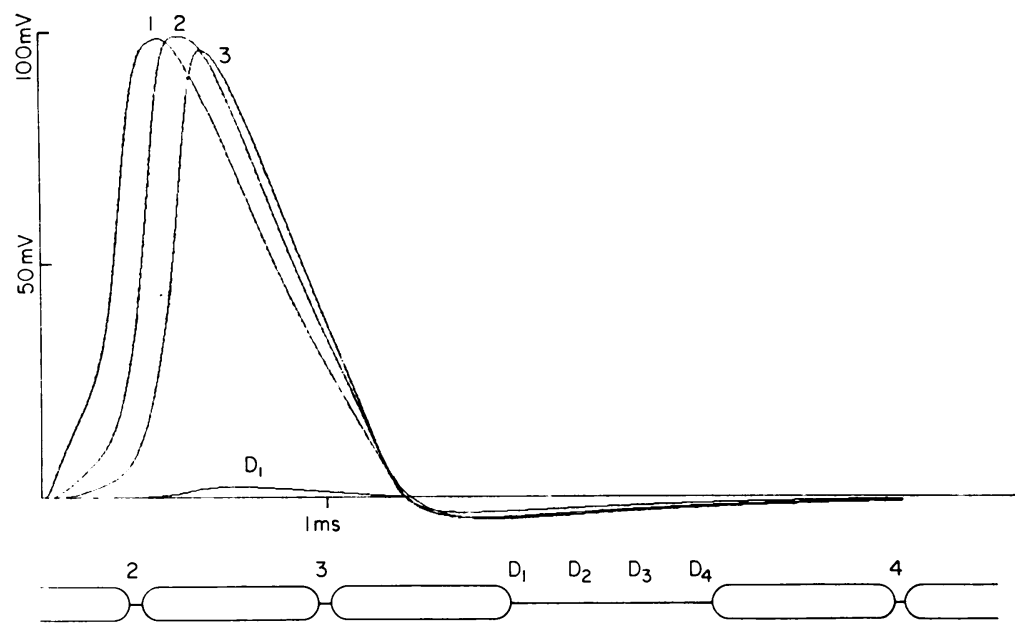

Fig. 1 Computed action potentials for a fibre focally demyelinated in the region from $D_{1}$ to $D_{4}$. The axon membrane in the demyelinated region of the fibres represented in Figs. 1 to 5 has the same specific membrane properties as nodal axolemma. For the fibre shown in this Figure, the internode proximal to the demyelinated region $\left(3-D_{1}\right)$ is of normal $(2000 \mu m)$ length. Despite the assumption of excitable membrane in the demyelinated region, conduction fails at point $D_{1}$ as a result of inadequate current density. In this and the following Figures, potentials from nodes $1-6$ and in the demyelinated region $\left(D_{1}-D_{4}\right)$ are shown; potentials at nodes $7-11$ are omitted for clarity. Schematic diagrams below the traces show fibre geometry in the vicinity of the demyelinated region; internode 1-2 and all internodes distal to node 4 are of normal length.

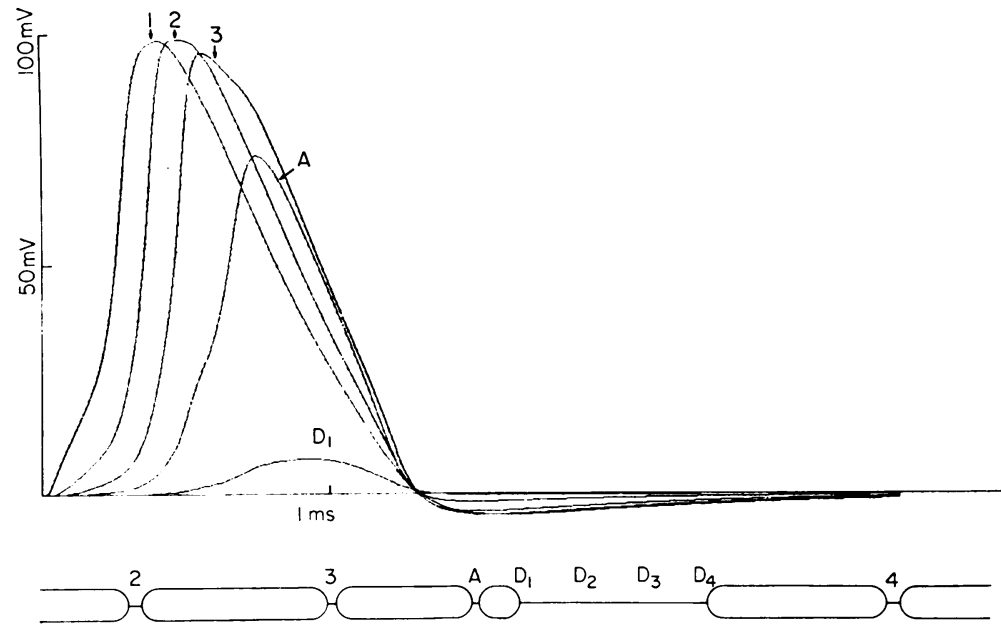

Fig. 2 Computed action potentials for a fibre similar to that shown in Fig. 1 , but with a single short internode $\left(A-D_{1} ; 400 \mu m\right)$ interposed proximal to the demyelinated region. There is conduction failure at point $D_{1}$. 

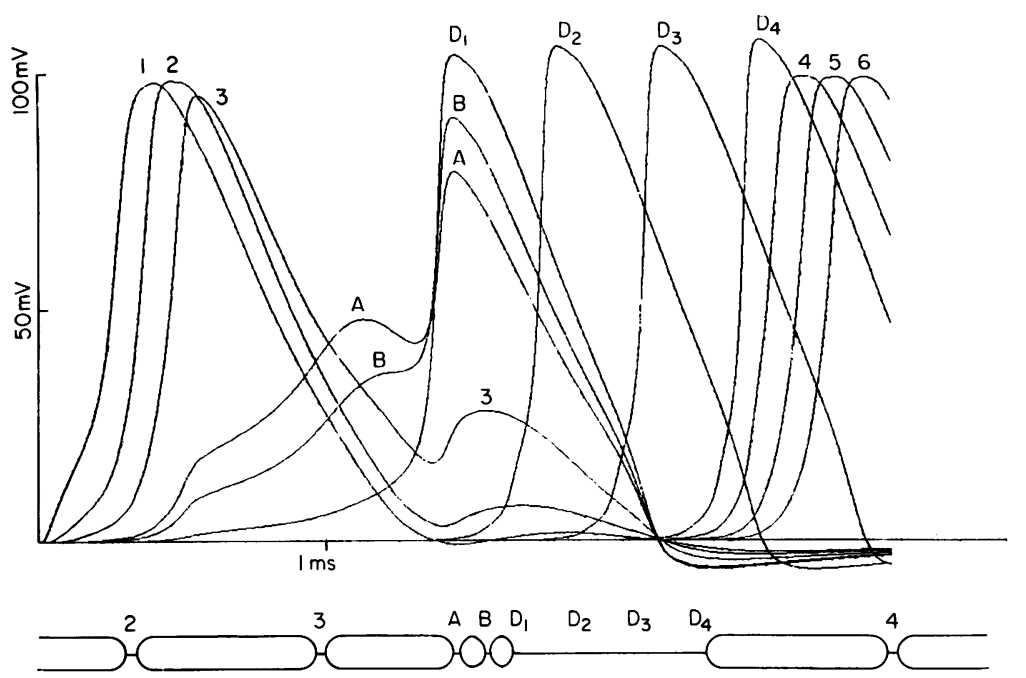

Fig. 3 Computed action potentials for a fibre similar to that shown in

Fig. 1, with two short internodes $\left(A-B, B-D_{1} ; 200 \mu m\right)$ interposed proximal to the demyelinated region. Under these conditions, the impulse invades the demyelinated region, and passes without interruption into the distal part (for example, nodes 4, 5, 6) of the fibre.

and then proceeds along the normally myelinated distal part of the fibre (nodes $4,5,6$, and subsequent nodes) at a normal conduction velocity $(19.7 \mathrm{~m} / \mathrm{s})$. In this case, nodes $A$ and $B$ and point $D_{1}$ fire almost synchronously. The delay from excitation at node 3 to excitation at point $D_{1}$ is $0.92 \mathrm{~ms}$.

A focally demyelinated fibre, with two $400 \mu \mathrm{m}$ internodes interposed before the demyelinated region, is shown in Fig. 4. Conduction again proceeds into and beyond the demyelinated region. In this case, the delay between excitation of node 3 and of point $D_{1}$ is $0.51 \mathrm{~ms}$, a value considerably shorter than in the previous simulation. Conduction velocity within the demyelinated region is, as in the previous simulation, approximately $1.64 \mathrm{~m} / \mathrm{s}$, and conduction proceeds at a normal conduction velocity in the distal myelinated internodes.

Figure 5 shows a still larger spacing of the nodes proximal to the demyelinated area; in this case the two proximal internodes closest to the demyelinated region are $600 \mu \mathrm{m}$ long. Conduction proceeds into, and past, the demyelinated zone, although invasion time (from excitation of node 3 to point $D_{1}$ ) is increased to $0.82 \mathrm{~ms}$. If length of the two internodes proximal to the demyelinated region is increased to $1000 \mu \mathrm{m}$, conduction failure occurs at point $\mathrm{D}_{1}$. Thus, the optimal value for internode distances proximal to the demyelinated region, in terms of minimising the time required for invasion of the demyelinated region, falls between $200 \mu \mathrm{m}$ and $600 \mu \mathrm{m}$, and internode lengths of more than $1000 \mu \mathrm{m}$ will not facilitate invasion of the demyelinated zone.

Since it is also possible (see Discussion) that specific membrane properties in demyelinated regions may be different from those at normal nodes, we examined conduction in focally demyelinated fibres, in which the axon membrane in the demyelinated region had properties of Hodgkin-Huxley (1952) membrane (see Table). Despite the much higher resistance than at nodal membrane, conduction again failed when the internodes proximal to the demyelinated region were of normal $(2000 \mu \mathrm{m})$ length. However, when the proximal internodes closest to the demyelinated region were reduced in length, as shown in Fig. 6, the impulse invaded, and passed, the demyelinated region. In this case, the delay from excitation of node 3 to excitation of point $D_{1}$ was $0.42 \mathrm{~ms}$, and conduction velocity in the demyelinated region was approximately equal to that for a uniform fibre with Hodgkin-Huxley membrane propertiesthat is $1.62 \mathrm{~m} / \mathrm{s}$. This simulation demonstrates that (a) impulses can propagate through, and beyond, regions of demyelination at which sodium channel density is significantly lower than at normal nodal membrane, but (b) such propagation may require a mechanism for impedance matching in the demyelinated zone, which in the present simulation was provided by reduction in internode length proximal to the site of demyelination. 

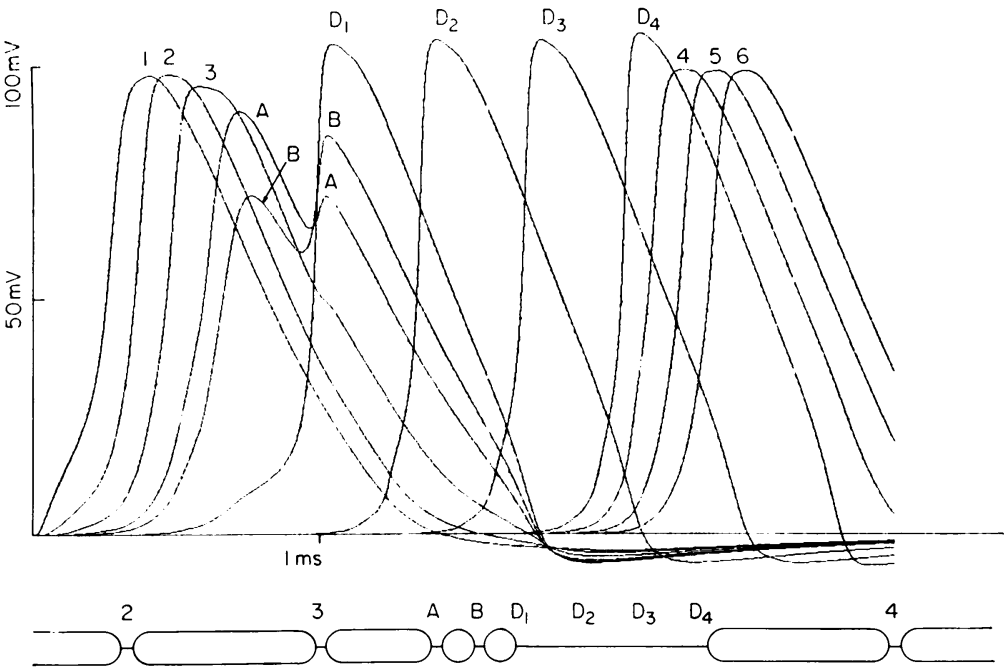

Fig. 4 Computed action potentials for a fibre with two $400 \mu \mathrm{m}$ internodes $\left(A-B, B-D_{1}\right)$ proximal to the demyelinated zone. Conduction again proceeds through the demyelinated region, but with a shorter latency than in Fig. 3.

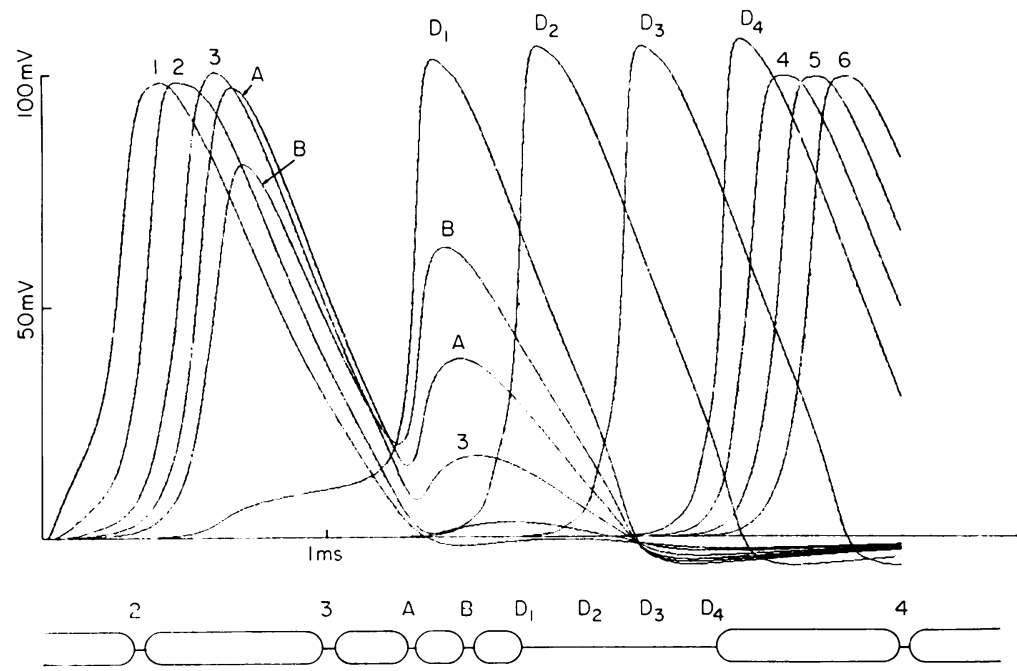

Fig. 5 Computed action potentials for a fibre with two $600 \mu$ m internodes $\left(A-B, B-D_{1}\right)$ proximal to the demyelinated zone. Conduction proceeds through the demyelinated region, but requires longer than for the fibre shown in Fig. 4. 


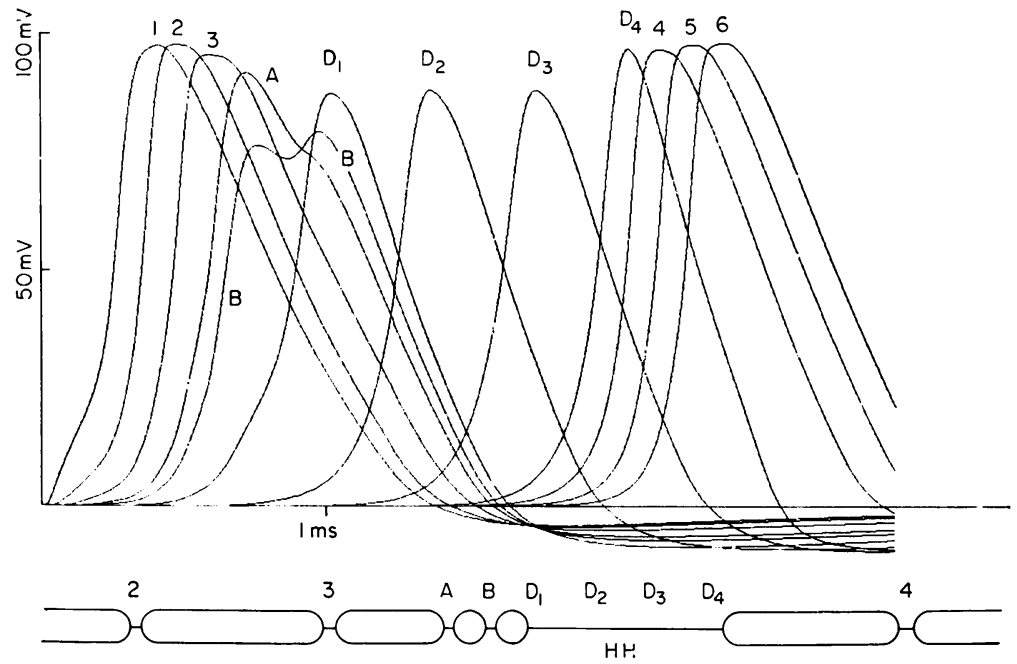

Fig. 6 Conduction past a demyelinated region $\left(D_{1}-D_{4}\right)$ in which the axon membrane has the characteristics of Hodgkin-Huxley membrane. Despite the lower sodium channel density than at nodes of Ranvier, conduction into, and beyond, the demyelinated region occurs. However, as in the simulations shown previously, uninterrupted conduction requires the presence of two short $(400 \mu \mathrm{m})$ internodes proximal to the demyelinated zone.

\section{Discussion}

The present results, like those of earlier studies (Koles and Rasminsky, 1972), indicate that impulse propagation in demyelinated fibres is sensitive to fibre geometry. The present findings further suggest that reduction in internode distance proximal to a demyelinated region may play a role in permitting action potentials to invade, and pass, the demyelinated area. The situation is similar to that described by Revenko et al. (1973) who showed that, because current density is inadequate, an impulse from a myelinated fibre will fail to invade a non-myelinated terminal, unless either the last internode is shorter than the rest, or the terminal has a reduced diameter. In the case of the present simulation, we assumed the presence of a sufficient density of sodium channels in the demyelinated region to support conduction, but nevertheless found that it was necessary to reduce the length of the two proximal internodes closest to the demyelinated region. The two final nodes then exhibited diphasic impulses and fired nearly synchronously, so that current density in the demyelinated region was increased not only as a result of the decreased capacitative and resistive current loss in the shortened internodes, but also as a result of increased current generation. This is similar to the observation by Goldstein and Rall (1974) and by Ramon et al. (1975) that at transitional regions along some inhomo- geneous non-myelinated axons close to zones of low safety factor, the action potential may be diphasic and will be generated simultaneously along large regions of the axon. The degree of internodal shortening required for invasion of the demyelinated region in our simulations was relatively modest, with reduction to approximately one-third of normal or less being required for propagation into and beyond the demyelinated region. Since safety factor is dependent on temperature (Davis and Jacobson, 1971; Rasminsky, 1973), it should be noted that the present simulations, like those of Koles and Rasminsky (1972), were carried out at $20^{\circ} \mathrm{C}$. Our simulations, like those of earlier workers (Koles and Rasminsky, 1972; Schauf and Davis, 1974), are based on the assumption that mammalian nodes of Ranvier exhibit specific membrane properties similar to those in amphibian nerve fibres. As noted by Koles and Rasminsky (1972), the available data suggest that this is not unreasonable. Our own studies (Moore et $a l ., 1978)$ indicate that conduction velocity in myelinated fibres is far more sensitive to internodal parameters than to the nodal description. Nevertheless, it should be noted that the precise degree of internodal shortening required will, of course, depend on the exact description of the myelin and of the membrane properties in the nodes and demyelinated area.

Our results are similar to those of Revenko et al. (1973) in indicating that increasing the resistivity of 
the demyelinated axon membrane did not effectively promote conduction into the demyelinated region. As in normal fibres (Moore et al., 1978), conduction properties in demyelinated fibres appear to be quite sensitive to internodal structure.

Internode distances are reduced, compared to those in peripheral fibres, in some preferminal fibres in the normal central nervous system (Waxman, 1970, 1975). At these sites, the changes in internode distances may permit axons to function as delay lines (Waxman, 1975) or may function so as to modulate invasion of axonal terminals (Waxman, 1972; Revenko et al., 1973).

Several ultrastructural studies have shown that internode distances may also be substantially reduced along central remyelinated axons (Suzuki et al., 1969; Gledhill et al., 1973). The present results suggest that the reduced internode distances might function so as to facilitate conduction past focally demyelinated regions. It should be emphasised in this context that several lines of evidence suggest that, in normal fibres, sodium channels are present at higher density at the nodes of Ranvier than in the internodes (Ritchie and Rogart, 1977; Waxman, 1977). An explicit assumption of the simulations shown in Figs. 1 to 5 is that normal nodal sodium channel densities develop at the newly formed nodes and over demyelinated regions of the axolemma. While there is, at present, little evidence directly bearing on the question of sodium channel density at newly formed nodes, there is some evidence for normal or near normal physiological function of newly formed nodes in remyelinated fibres (see Rasminsky, 1978). It should be noted, however, that invasion of a demyelinated region with lower values of sodium conductance than at normal nodes is also possible if there is a reduction in length of the proximal internodes (see Fig. 6). Cytochemical studies in dystrophic mice suggest, in fact, that ionic channel densities along the amyelinated regions of ventral root axons may be lower than at normal nodes of Ranvier (Waxman et al., 1978).

Longitudinal current analyses, which show a transition from saltatory to continuous conduction along some ventral root fibres demyelinated with diphtheria toxin (Bostock and Sears, 1976), and along some amyelinated ventral root fibres in dystrophic mice (Rasminksy et al., 1977), have not, to date, resolved the question of whether there are short internodes at the transitional zones along these axons. As noted by Rasminsky et al. (1977), resolution of the recording techniques was such that shorter internodes would not be detected. It is possible that some other mechanism facilitates invasion of nonmyelinated zones in these axons, or, as suggested by Fig. 5, that only small changes in fibre geometry are required. However, reduced internode distances were observed histologically in the amyelinated axons (Rasminsky et al., 1977).

Our previous studies (Brill et al., 1977) showed that substantial reductions in internode distance are associated with decreased axonal conduction velocity. Several authors, however, have suggested that clinical deficits in multiple sclerosis may be primarily related to conduction failure, rather than to slowing of conduction (see, for example, Rasminsky, 1973; Halliday and McDonald, 1977). It has been suggested that the demyelinated axon membrane may exhibit structural plasticity (Ritchie and Rogart, 1977; Waxman, 1977). If this is the case, reduction in internode distances along remyelinated fibres might play a role in promoting functional recovery as a result of facilitation of uninterrupted transmission along affected axons, despite the prolonged conduction time. As in the normal central nervous system (Waxman, 1975), matching of internode distances to functional requirements may be of considerable importance. This conclusion may be relevant to the search for agents which initiate, and control, remyelination in multiple sclerosis.

This work was supported in part by grants from the US National Multiple Sclerosis Society (RG-1133A-1), the National Institute of Health (NS-12307, RR-05479), and the Health Sciences Fund (78-10). Dr Waxman is the recipient of a Research Career Development Award (K04-NS-00010) from the National Institute of Neurological and Communicative Disorders and Stroke. We thank Professor J. Y. Lettvin for helpful discussions.

\section{References}

Bostock, H., and Sears, T. A. (1976). Continuous conduction in demyelinated mammalian nerve fibres. Nature, 263, 786-787.

Brill, M. H., Waxman, S. G., Moore, J. W., and Joyner, R. W. (1977). Conduction velocity and spike configuration in myelinated fibres: computed dependence of internode distance. Journal of Neurology, Neurosurgery, and Psychiatry, 40, 769 774.

Davis, F. A., and Jacobson S. (1971). Altered thermal sensitivity in injured and demyelinated nerve. Journal of Neurology, Neurosurgery, and Psychiatry, 34, 551-561.

Dodge, F. A., and Cooley, J. W. (1973). Action potential of the motoneuron. IBM Journal of Research and Development, 17, 219-229.

FitzHugh, R. (1962). Computation of impulse initiation and saltatory conduction in a myelinated nerve fiber. Biophysical Journal, 2, 11-21.

Ghatak, N. R., Hirano, A., Lijtmaer, H., and Zimmerman, H. M. (1974). Asymptomatic demyelinated 
plaque in the spinal cord. Archives of Neurology (Chicago), 30, 484-486.

Gledhill, R. F., Harrison, B. M., and McDonald, W. I. (1973). Pattern of remyelination in the CNS. Nature, 244, 443-444.

Goldstein, S. S., and Rall, W. (1974). Changes of action potential shape and velocity for changing core conductor geometry. Biophysical Journal, 14, 731-757.

Halliday, A. M., and McDonald, W. I. (1977). Pathophysiology of demyelinating disease. British Medical Bulletin, 33, 21-27.

Halliday, A. M., McDonald, W. I., and Mushin, J. (1973). Visual evoked response in diagnosis of multiple sclerosis. British Medical Journal, 4, 661664.

Hodgkin, A. L., and Huxley, A. F. (1952). A quantitative description of membrane current and its application to conduction and excitation in nerve. Journal of Physiology (London), 117, 500-544.

Khodorov, B. I., Timin, Y. N., Vilenkins S. Y., and Gulko, F. B. (1969). Theoretical analysis of the mechanisms of conduction of nerve impulse over an inhomogeneous axon: conduction through a portion with increased diameter. Biofizika, 14, 304 315.

Koles, Z. J., and Rasminsky, M. (1972). A computer simulation of conduction in demyelinated nerve fibres. Journal of Physiology, 227, 351-364.

Moore, J. W., Ramon, F., and Joyner, R. W. (1975). Axon voltage-clamp simulations I. Methods and tests. Biophysical Journal, 15, 11-24.

Moore, J. W., Joyner, R. W., Brill, M. H., Waxman, S. G., and Najar-Joa, M. (1978). Simulations of conduction in myelinated fibres: relative sensitivity to changes in nodal and internodal parameters. Biophysical Journal, 21, 147-160.

Namerow, N. S., and Thompson, L. R. (1969). Plaques, symptoms, and the remitting course of multiple sclerosis. Neurology (Minneapolis), 19, 765-774.

Parnas, I., Hochstein, S., and Parnas, H. (1976). Theoretical analysis of parameters leading to frequency modulation along an inhomogeneous axon. Journal of Neurophysiology, 39, 909-923.

Ramon, F., Joyner, R. W., and Moore, J. W. (1975). Propagation of action potentials in inhomogeneous axon regions. Federation Proceedings, 34, 13571363.
Rasminsky, M. (1973). The effects of temperature on conduction in demyelinated single nerve fibers. Archives of Neurology (Chicago), 28, 287-292.

Rasminsky, M. (1978). Physiology of conduction in demyelinated axons. In Physiology and Pathobiology of Axons, pp. 361-376. Edited by S. G. Waxman. Raven Press: New York.

Rasminsky, M., and Sears, T. A. (1972). Internodal conduction in undissceted demyelinated nerve fibres. Journal of Physiology, 227, 323-350.

Rasminsky, M., Kearney, R. E., Aguayo, A. J., and Bray, G. M. (1977). Conduction of nervous impulses in spinal roots and peripheral nerves of dystrophic mice. Brain Rescarch. In press.

Revenko, S. V., Timin, Ye. N., and Khodorov, B. I. (1973). Special features of the conduction of nerve impulses from the myelinized part of the axon into the non-myelinated terminal. Biofizika, 18, 10741078.

Ritchie, J. M., and Rogart, R. B. (1977). The density of sodium channels in mammalian myelinated nerve fibres and the nature of the axonal membrane under the myelin sheath. Proceedings of the National Academy of Sciences, 74, 211-215.

Schauf, C. L., and Davis, F. A. (1974). Impulse conduction in multiple sclerosis: a theoretical basis for modification by temperature and pharmacological agents. Journal of Neurology, Neurosurgery. and Psychiatry, 37, 152-161.

Suzuki, K., Andrews, J. M., Waltz, J. M., and Terry, (ষ R. D. (1969). Ultrastructural studies of multiple sclerosis. Laboratory Investigation, 20, 444-454.

Waxman, S. G. (1970). Closely spaced nodes of Ranvier in the teleost brain. Nature, 227, 283-284.

Waxman, S. G. (1972). Regional differentiation of the axon: a review with special reference to the concept $\frac{0}{\partial}$ of the multiplex neuron. Brain Research, 47, 269 288.

Waxman, S. G. (1975). Integrative propertics and design principles of axons. International Review of Neurobiology, 18, 1-40.

Waxman, S. G. (1977). Conduction in myelinated, unmyelinated, and demyelinated fibres. Archives of Neurology (Chicago), 34, 585-589.

Waxman, S. G., Bradley, W. G., and Hartwieg, E. A. (1978). Organization of the axolemma in amyelinated axons: a cytochemical study in dy/dy dystrophic mice. Proceedings of the Royal Society. Series $B$. In press. 